\title{
A Combined PWM and AEM-Based AC Voltage Controller for Resistive Loads
}

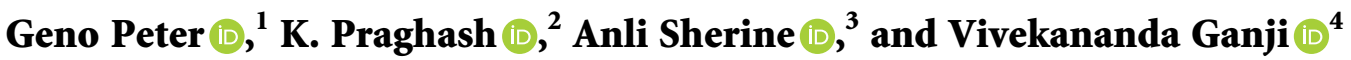 \\ ${ }^{1}$ CRISD, School of Engineering and Technology, University of Technology Sarawak, Sibu, Malaysia \\ ${ }^{2}$ Department of Electronics and Communication Engineering, Koneru Lakshmaiah Education Foundation, Guntur, India \\ ${ }^{3}$ School of Computing and Creative Media, University of Technology Sarawak, Sibu, Malaysia \\ ${ }^{4}$ Department of Electrical and Computer Engineering, Debre Tabor University, Debre Tabor, Ethiopia \\ Correspondence should be addressed to Vivekananda Ganji; drvivek@bhu.edu.et
}

Received 7 January 2022; Accepted 20 January 2022; Published 14 February 2022

Academic Editor: Albert Alexander Stonier

Copyright $\odot 2022$ Geno Peter et al. This is an open access article distributed under the Creative Commons Attribution License, which permits unrestricted use, distribution, and reproduction in any medium, provided the original work is properly cited.

AC voltage controller using PWM technique integrated with equal area digital modulation technique and connected to resistive loads is discussed in this paper. The proposed technique reduces the harmonics in the lower order significantly and improves the power factor compared to the existing conventional line commutated voltage controllers. The voltage and current waveforms are smoothened; therefore, a sinusoidal nature is achieved. The power factor is considerably improved at the low output voltage range when compared to existing methods. The capabilities of the proposed technique are computed mathematically and simulation results are compared with the existing methods.

\section{Introduction}

The voltage and current waveforms delivered to the customer should satisfy the different international standards, thereby ensuring quality power to different domestic and industrial applications. Conventional outage management practices in the distribution system are tedious and complex due to long time taken in locating the fault. Emerging smart technologies and various cloud services offered could be utilized and integrated into the power industry to enhance the overall process, especially in the fault monitoring and normalizing fields in the distribution system. The voltage waveform may experience a sag, swell, surges, and harmonics which causes the sinusoidal waveform to be distorted. AC voltage controllers with line frequency control are commonly used as it does not require any sophisticated commutation circuit for switching and stable control. It is used in various applications such as heater room control, induction motor starting, fan, and pump speed control [1]. The output voltage control is obtained by controlling the switching phase. Though line commutated AC voltage controllers are economic in design, it suffers from low power factor, thereby creating harmonics in the input current waveform [2]. Phase angle control (PAC) method has a poor input power factor, and the power flow is not continuous even for resistive loads. This method when used for electric drive speed control causes pulsating torque and harmonics on the input frequency. The power factor of such controllers is generally very low when the output voltage is decreased which is at a higher firing angle [3]. Force commutated AC voltage converters show a significant improvement in terms of efficiency as the input power factor is improved and the harmonics have reduced very significantly [4]. When compared to PAC method, control methods such as symmetric angle control (SAC) employing forced commutation produce better results in the form of reduced THD and improved PF during in AC-AC conversion. Using a new technique called area equalization method reduction in THD, complete elimination of lower order harmonics and power factor improvement can be achieved [5].

For the proposed technique, Matlab-Simulink Real-Time Target Windows (RTW) provided by Math Works Inc., is used for half-wave symmetry, multiple pulses, and direct PWM technique wherein several pulses per half cycle $(m)$ are 
generated under the integrated development environment [6]. Continuously variable output voltage is obtained by squaring off the actual output voltage with the target output voltage by using these pulses. For different values of $m$, the chopped output voltage waveform is analyzed for its harmonic content. For evaluation, the SAC method has been considered, and while doing so, the harmonics profile is found to be improved with the proposed digital strategy for certain values of $m$. The filtering requirements are reduced to a minimum as higher-order harmonics are pushed away from the fundamental frequency towards the sampling frequency for the entire output voltage range. Thus, improvement of THD and improved power factor at highfrequency chopping mode (more $\mathrm{m}$ ) facilitate easy filtration at a lower cost [7].

In a power electronic circuit if the switches operate in a discontinuous fashion resulting in the generation of harmonics, lagging power factor occurs on the input side of the converter when the firing angle is delayed. The development in the power electronics design technology has paved the way to improve the efficiency of the converters by reducing the switching losses and the harmonics. AC line commutated controllers can be operated in chopped mode, hence providing a better output voltage control. The output voltage of the converter can be controlled by the duty cycle of the controlled switches as the output and input voltages are chopped into segments. Sinusoidal output voltage and current are obtained when using the PWM technique as the power factor is improved and the harmonics at lower order are reduced considerably. In electric drives, switching control method is used which heats up the induction motor due to the switching losses and harmonics present [8]. The power quality issue can occur when the renewable energy sources deliver power to the grid. Insulated gate bipolar junction transistor (IGBT)-based pulse with modulation technique is used to improve the power quality [14]. PWM techniques are used to eliminate the harmonics of lower order so that the output voltage and current waveform get improved. PWM technique can be implemented in power electronics converters using a square wave, sampled wave, and sinusoidal wave, and the designer makes the choice [15].

In the proposed method, the PWM technique is enhanced using the equal area criteria method. The actual output is made equal as the sampling time for both the output voltage and the pulse area is the same. The switching pulses are generated by the control circuit irrespective of the technique used to control the converter operation [16]. The simulated proposed method results are compared with the existing methods, and the main goal is to reduce the total harmonic distorting using the enhanced method. The power factor is considerably improved at the low output voltage range when compared to symmetric angle control (SAC) method using this technique.

\subsection{Digital Control Strategies for AC Voltage Controllers}

1.1.1. Symmetrical Angle Control. The single-phase circuit bidirectional topology of an AC voltage regulator is shown in Figure 1. It consists of a pair of metal oxide semiconductor field-effect transistors (MOSFET) connected in parallel and a diode in series so that the reverse voltage can be blocked [9]. Bidirectional power transfer prevents the problem of DC input current, and the topology dictates the use of blocking the voltage in either direction by the switches and controlling the reverse and forward conduction of the current.

Both S1 and S2 are forward biased during positive and negative half-cycles and made to conduct for the same conduction angle $(\delta)$, from $(\pi / 2-\delta / 2)$ to $(\pi / 2+\delta / 2)$ and $(3 \pi / 2-\delta / 2)$ to $(3 \pi / 2+\delta / 2)$, respectively, and the concept of the SAC method is shown in Figure 2.

The conceptual diagram of the SAC method for half-sine wave is shown, and the firing angle is chosen at a particular value so that it has to provide a maximum conduction period, so that the load can get maximum load voltage with reduced harmonics, thus enhancing continuous conduction mode over a long period of time. The input voltage and output voltage waveforms along with gating signals are shown in Figure 3.

If $V_{s}=\sqrt{ } 2 V_{s} \sin \omega t$ is the input voltage, and the pulse widths for S1 and S2 are equal, the output voltage RMS value is calculated as

$$
\begin{aligned}
& V_{0}=\left[\frac{2}{\pi} \int_{\pi-\delta / 2}^{\pi+\delta / 2} V_{m}^{2} \sin ^{2} \omega t d(\omega t)\right]^{1 / 2}, \\
& V_{0}=\frac{V_{m}}{\sqrt{2}}\left[\frac{1}{\pi}(\delta+\sin \delta)\right]^{1 / 2} \text { Volts. }
\end{aligned}
$$

Thus, by varying $\delta$ from $\pi$ to 0 , the RMS output voltage can be varied from RMS input voltage $V_{s}$ to 0 .

\section{Proposed Area Equalization Method}

In the SAC method, the lower order harmonics are dominant and the THD increases with $\delta$, as it is varied from $\pi$ to 0 and is high for the minimum output voltage [10].

The AEM is a direct PWM strategy suitable for singlephase AC voltage regulators. In this method, switching is done in such a way that the integrated area of the target output voltage waveform over the carrier interval is the same as the integrated area of the actual output voltage waveform, i. e., each area or pulse in the actual output waveform for a duration is equated to the region under the sampled target waveform for the same duration and hence the name area equalization method (AEM) [11]. The concept of the proposed AEM is illustrated in Figure 4. To equate the target output voltage with the actual output voltage, the area under the target output voltage is also divided into the same number of samples $(m)$ per half-cycle as in the actual output.

The lower and upper limits for any sample in AEM lie between $\left((k-1) \pi / m+X_{o i}-\delta_{k} / 2\right)$ and $((k-1) \pi / m+$ $\left.X_{o i}+\delta_{k} / 2\right)$, and the conceptual diagram shown in Figure 4 explains the AEM concept. As pointed out earlier, AEM is a direct PWM method and the pulse width $(\delta \mathrm{k})$ is determined by equating the two regions shown in Figure 4. 


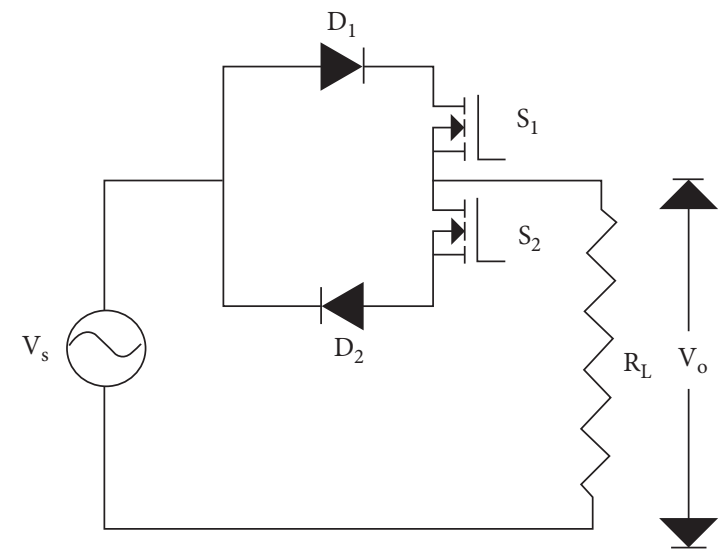

FIGURE 1: Single-phase AC voltage regulator power circuit.

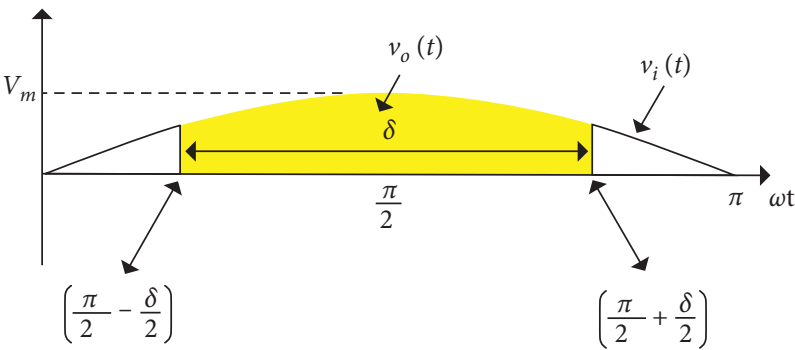

FIgure 2: Conceptual diagram for the SAC method.

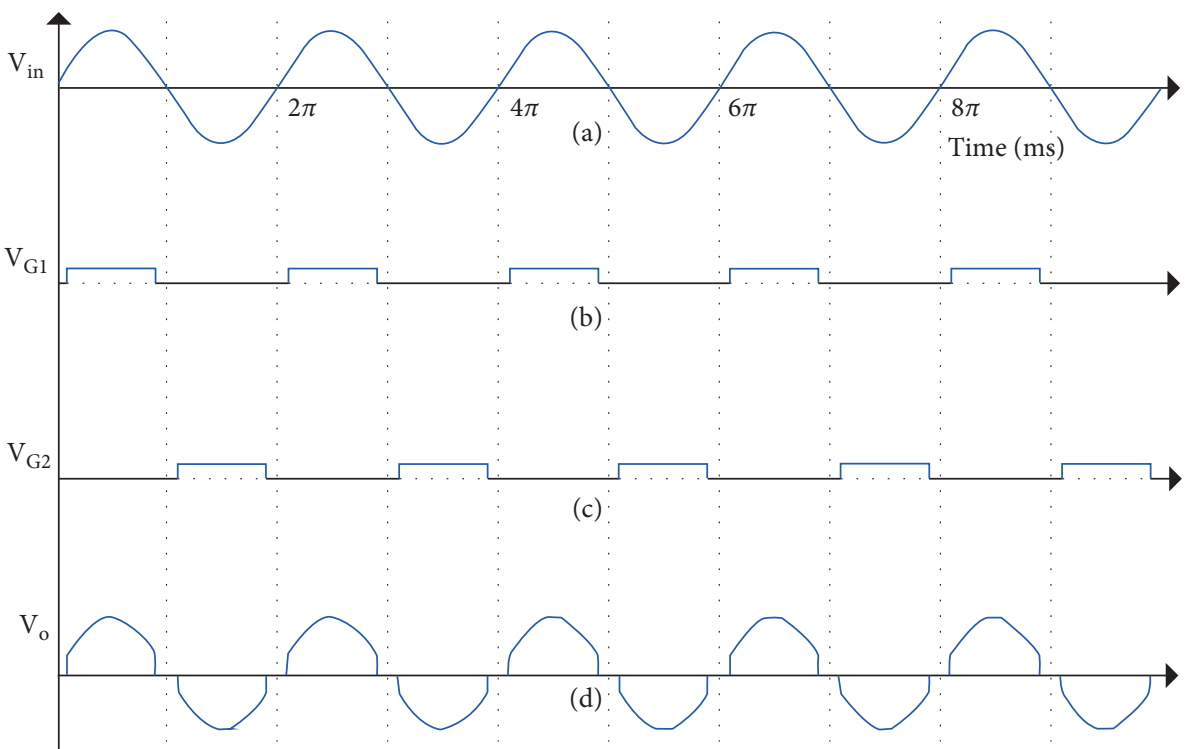

FIGURE 3: SAC method. (a) Input voltage waveform. (b) and (c) gating signals for S1 and S2. (d) Output voltage waveform. 


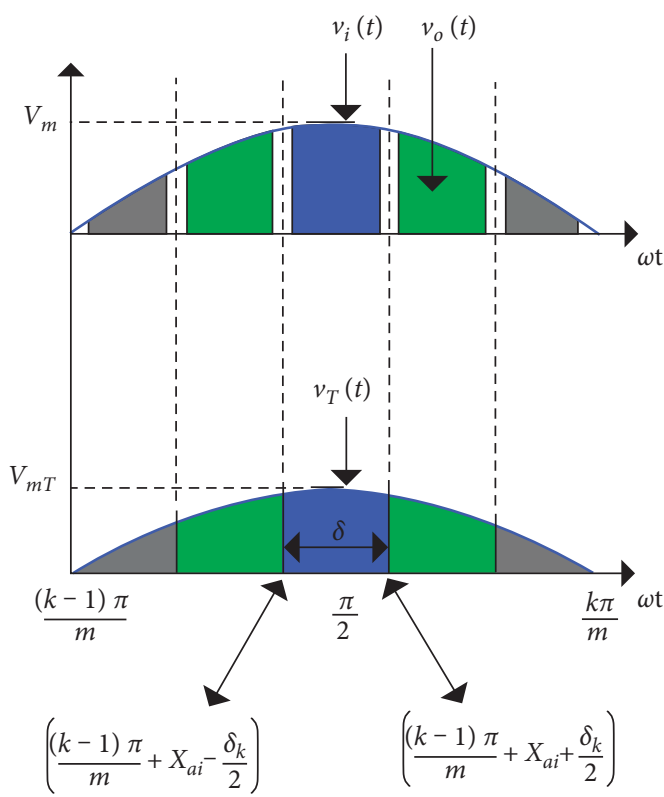

Figure 4: Area equalization method (AEM).

$$
A_{k}=V_{m T}\left[\cos \left(\frac{(k-1) \pi}{m}\right)-\cos \left(\frac{k \pi}{m}\right)\right],
$$

where $A_{k}$ is the area of the $k^{\text {th }}$ sample of the target voltage, $V_{m T}$ is the maximum value of the target voltage, $m$ is the number of samples per half cycle, and $k$ is the $k^{\text {th }}$ sample; $k=1,2,3, \ldots \ldots \ldots$.

$$
A_{k o}=\int_{\left((k-1) \pi / m+X o i-\delta_{k} / 2\right)}^{\left((k-1) \pi / m+X o i+\delta_{k} / 2\right)} V_{m} \sin \omega t d(\omega t),
$$

where $A_{k o}$ is the area of the $k^{\text {th }}$ sample in the actual output, $V_{\mathrm{m}}$ is the value of the maximum input voltage, and $\partial$ is the conduction angle.

$$
\begin{aligned}
A_{k o} & =2 V_{m}\left[\sin (k-0.5) \frac{\pi}{m} \sin \left(\frac{\delta_{k}}{2}\right)\right], \\
A_{k} & =A_{\text {outputk }} \\
V_{m T}\left[\cos \left(\frac{(k-1) \pi}{m}\right)-\cos \left(\frac{k \pi}{m}\right)\right] & =2 V_{m}\left[\sin (k-0.5) \frac{\pi}{m} \sin \left(\frac{\delta_{k}}{2}\right)\right], \\
\frac{\delta_{k}}{2} & =\sin ^{-1}\left[\frac{V_{m T}[\cos ((k-1) \pi / m)-\cos (k \pi / m)]}{2 V_{m} \sin (k-0.5) \pi / m}\right] .
\end{aligned}
$$

The value of $\delta_{\mathrm{k}}$ obtained from (5) decides the pulse positioning to satisfy the AEM concept.

$$
\begin{aligned}
& V_{0}=\left[\frac{1}{\pi} \int_{0}^{\pi} V_{m}^{2} \sin ^{2} x \mathrm{~d} x\right]^{1 / 2} \\
& V_{0}=\left(\sum_{k=1}^{m}\left(\frac{V_{m}^{2}}{2 \pi}\left[\delta_{k}-\frac{1}{2}\left\{\sin \left[2\left(\frac{(k-1) \pi}{m}+X o i+\frac{\delta_{k}}{2}\right)\right]-\sin \left[2\left(\frac{(k-1) \pi}{m}+X o i-\frac{\delta_{k}}{2}\right)\right]\right\}\right]\right)\right)^{1 / 2} .
\end{aligned}
$$


The output voltage and input current contain harmonics and need Fourier analysis to decide the magnitude of the fundamental THD and the individual harmonic distortion. The analysis is carried out in the same way as it is done for the SAC method. The theoretical Fourier analysis removes the harmonics at higher order completely, and therefore, the power factor correction gets improved significantly and the harmonic distortion is reduced [12]. Figures 5-7 show the input voltage waveform, gate current pattern, and the output voltage measured at the load terminals.

The PWM technique for AC voltage controllers is completely different as the input voltage waveform is sliced numerously in each cycle while switching the converter. The DC voltage and the carrier wave which is triangular in nature are compared to get the switching signal. It is seen that the switching of the converter occurs at the point of interaction. The main frequency must be less than the converter switching frequency. Duty cycle of any converter is the ratio of the ON times to the completion of the switching cycle. The Simulink analysis for the proposed method is shown as follows.

\section{Simulink and Harmonic Analysis for SAC}

3.1. SAC Simulink Methodology. Using Matlab 7.1-Simulink platform, the AC voltage regulator shown in Figure 1 employing the SAC method is simulated.

The input voltage is $230 \mathrm{~V}, 50 \mathrm{~Hz}$, and the load resistance is $R L=110 \Omega$. Figure 3 shows the sample output voltage and input current waveforms along with their harmonic spectrum for $\delta=15.66$ corresponding to a low output voltage of $40 \mathrm{~V}$. A sample output of $40 \mathrm{~V}$ is chosen as the idea to study and describe the power quality and the PF in the low output voltage range [13].

Similarly, the sample output voltage and input current waveforms along with their harmonic spectrum for $\delta=55$ are shown in Figure 8 corresponding to a low output voltage of $130 \mathrm{~V}$.

As the load is resistive, the output voltage and current at the input side are in phase as well as the harmonics at lower order are dominant as shown in Figure 9, in the spectrum for $\delta=15.66$. The lower order harmonics are relatively less dominant for $\delta=55$ as shown in Figure 10. The harmonics at lower order is presiding in both the output voltage and input current waveforms which is objectionable, particularly at reduced output voltage levels $[11,12]$. The SAC output voltage is as shown in Figure 11.

Table 1 compares the theoretical and simulation results of the SAC method in terms of the fundamental RMS voltage. The target fundamental RMS voltage is $40 \mathrm{~V}$ with $\delta=15.66$ and the theoretical value of the fundamental voltage calculated using equations stands at $39.7718 \mathrm{~V}$ while the simulation yields $40.1 \mathrm{~V}$. While the fundamental voltage h1 is calculated as \% of target voltage, the individual harmonics $h_{\mathrm{a} 3}$ to $h_{\mathrm{a} 25}$ are calculated as the \% of fundamental voltage $\left(h_{\mathrm{a} 1}\right)$ using equations (4) and (6). The lower order harmonics are thus found to be dominant as seen in Table 1. Simulation results also substantiate the theoretical predictions. The multiples of the fundamental frequency are called voltage or current harmonics. The first harmonic is the fundamental component of the sinusoidal waveform. It is the base frequency on which periodically the waveform is built. Harmonics are caused because of the nonlinear loads, and the load which draws current proportional to the voltage fed from the input does not cause much implication to the power quality as the impedance is constant during the sinusoidal cycle.

\section{Simulink and Harmonic Analysis for AEM}

The power circuit model shown in Figure 1 is simulated again using Matlab-Simulink with the AEM concept applied to it. As seen in Figures 12(a) and 12(b), the proposed AEM provides a better power quality spectrum and relatively improved PF at reduced output voltage levels, the same target output voltage of $40 \mathrm{~V}$ with $\delta=31.18$ for AEM is chosen for the sake of comparison. AEM is one of the PWM methods, and we consider $m=10$ as the pulses per half cycle for our analysis. The simulated output voltage and input current waveforms are as shown in Figures 5-7, respectively.

It is evident from the spectrum that, the fundamental component is maintained at $100 \%$, and the lower order harmonics ranging from $h_{3}$ to $h_{17}$ are completely eliminated while $h_{19}$ and $h_{21}$ are dominant around. Still better results are obtained for higher voltages and such results are clearly shown in Figures 13(a) and 13(b). Table 2 describes the variation of harmonic order voltage content as the percentage of target fundamental output voltage of $40 \mathrm{~V}$.

\section{Comparative Analysis of SAC and AEM}

The distortion factor of individual harmonics as a percentage of the fundamental voltage also proves that the proposed AEM is superior to the SAC method as the harmonics $h_{3}$ to $h_{17}$ and $h_{23}$ and $h_{25}$ are only a fraction of the fundamental voltage. Though $h_{19}$ and $h_{21}$ are dominant, the filtering requirements are less as they are pushed far away from the fundamental frequency.

Total harmonic reduction places a vital role in improving power factors. However, the decrease in THD will increase the overall performance of the power factor for SAC and AEM as shown in Table 3. The comparative harmonic distortion factor for SAC and AEM is shown in Table 4.

The variations in PF with the input current for the SAC method and AEM with $m=11$ are shown in Figure 14. It is evident that the more the value of $m$ is, the better the PF will be, especially at the low output voltage range. From Figure 15 , it is evident that the power factor varies with " $m$ ".

Figure 16 shows the variation in THD with the fundamental target output voltage. This discloses that the SAC method has rich lower order harmonics and poor THD whereas the proposed AEM not only reduces the THD but also completely gets rid of the harmonics at lower order at the low target output voltage range for increasing samples $m$ as evident from Figure 17 [13]. It is clear that in the SAC method, the lower order harmonics are leading and dominant and the individual harmonic numbers are progressively decreasing whereas the harmonics of lower order are 


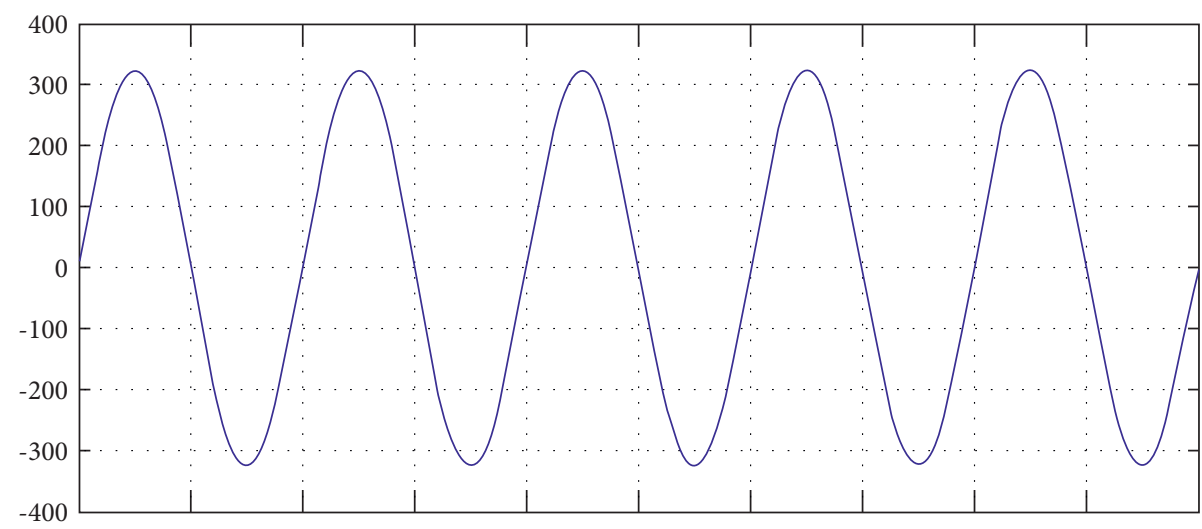

Figure 5: Input voltage waveform.
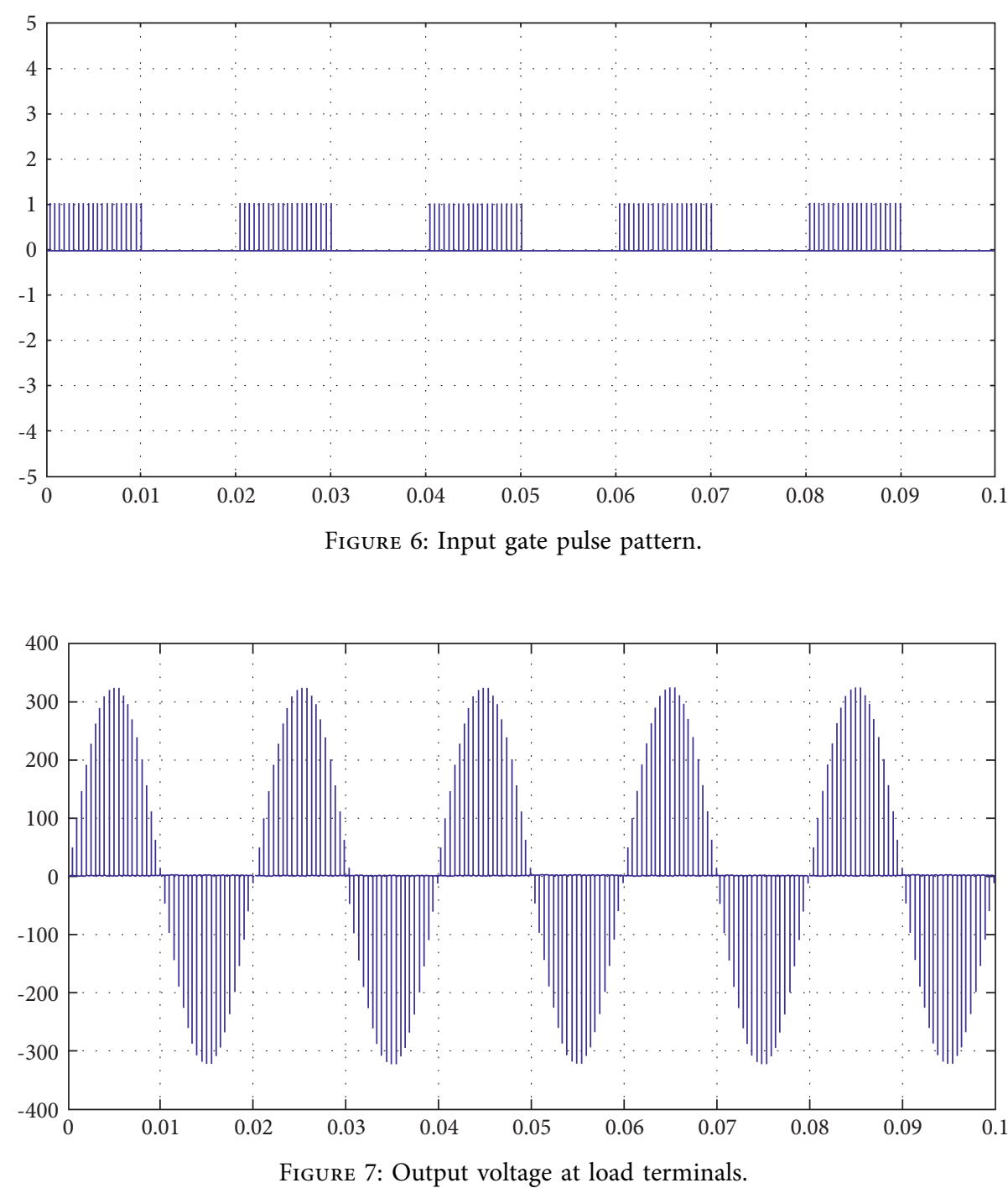

absolutely absent in AEM. At the same time, harmonics $h_{19}$ and $h_{21}$ are dominant only around, and hence, they can be filtered out using a low pass filter.
Figure 18 substantiates the concept that the increasing value of $m$ decides the placement of dominant harmonics in the high-frequency band for minimum filter requirements. 


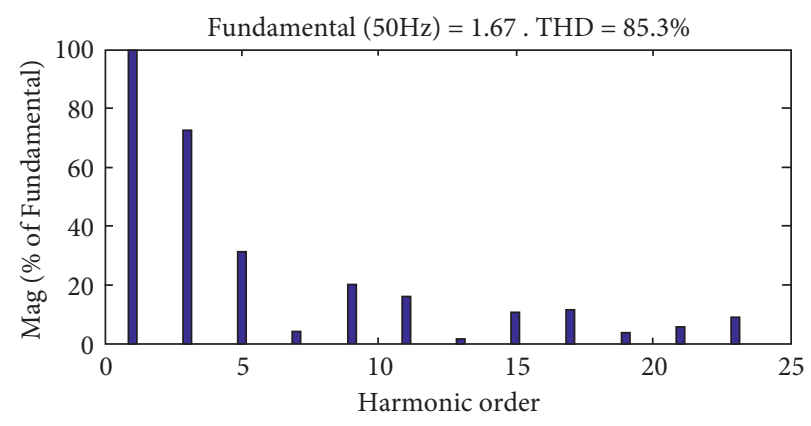

FIGURE 8: Harmonic spectrum for $\delta=55$.

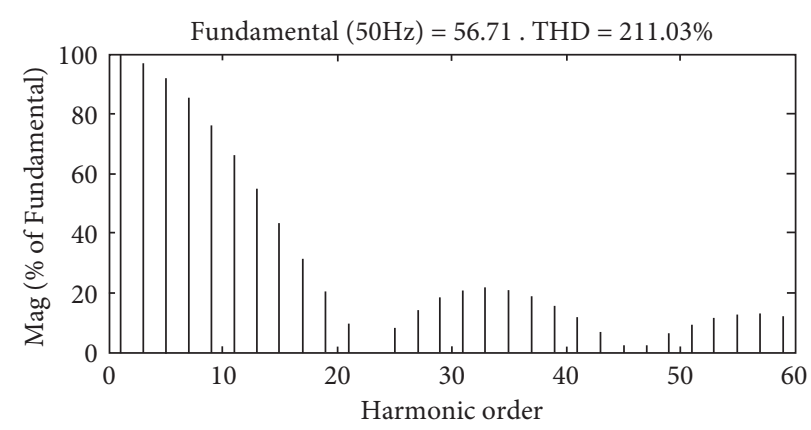

Figure 9: Harmonic spectrum for $\delta=15.66$.

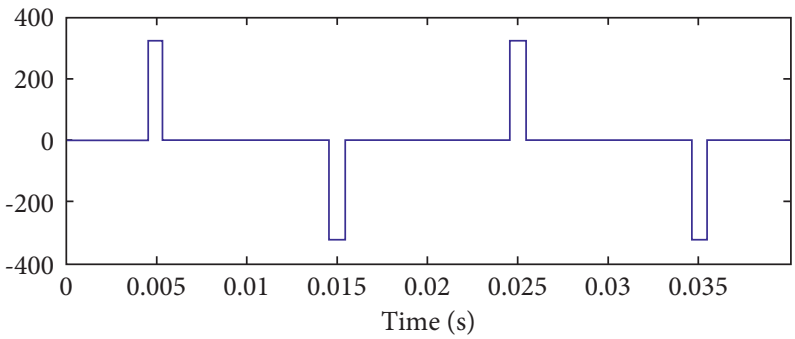

Figure 10: Input current for SAC.

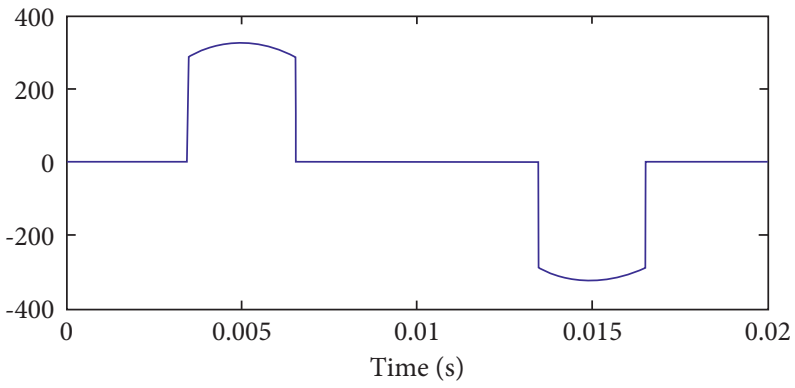

FIGURE 11: SAC output voltage. 
TABLE 1: Comparison of individual harmonics as $\%$ of the fundamental voltage.

\begin{tabular}{|c|c|c|}
\hline \multirow{2}{*}{ Harmonic order } & \multicolumn{2}{|c|}{ Fundamental RMS output voltage } \\
\hline & Theoretical & Simulation \\
\hline$h_{\mathrm{a} 1}$ & $39.7718(100 \%)$ & $40.1(100 \%)$ \\
\hline$h_{\mathrm{a} 3}$ & 97.53152 & 96.31464 \\
\hline$h_{\mathrm{a} 5}$ & 96.26102 & 91.53594 \\
\hline$h_{\mathrm{a} 7}$ & 85.7326 & 84.64122 \\
\hline$h_{\mathrm{a} 9}$ & 76.92234 & 75.94786 \\
\hline$h_{\mathrm{a} 11}$ & 66.65723 & 65.80855 \\
\hline$h_{\mathrm{a} 13}$ & 55.37655 & 54.66411 \\
\hline$h_{\mathrm{a} 15}$ & 43.554 & 42.99067 \\
\hline$h_{\mathrm{a} 17}$ & 31.67204 & 31.24671 \\
\hline$h_{\mathrm{a} 19}$ & 20.19883 & 19.90832 \\
\hline$h_{\mathrm{a} 21}$ & 9.557332 & 9.416334 \\
\hline$h_{\mathrm{a} 23}$ & 0.140099 & 0.1058 \\
\hline$h_{\mathrm{a} 25}$ & 7.778001 & 7.705878 \\
\hline
\end{tabular}

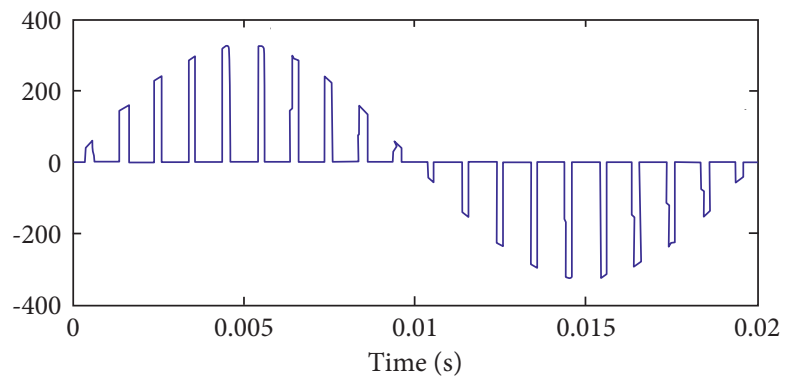

(a)

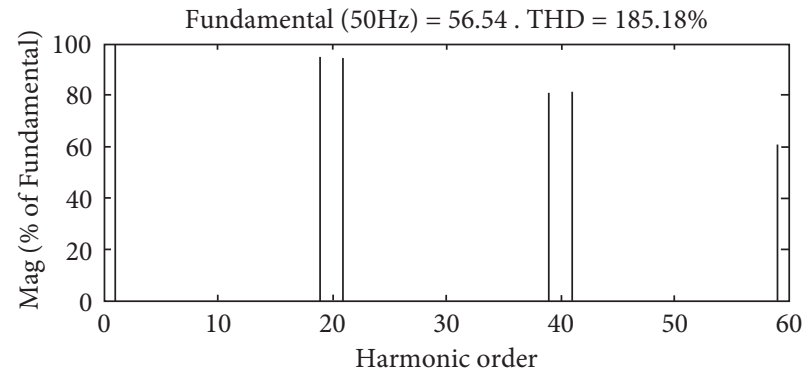

(b)

Figure 12: (a, b) AEM output voltage and harmonic spectrum for $\delta=31.18$.

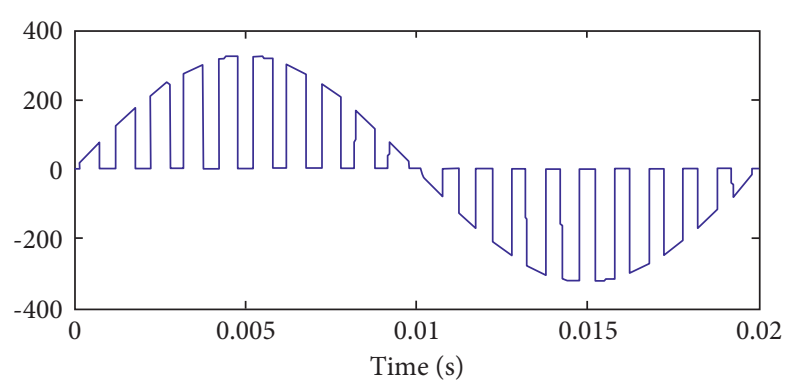

(a)

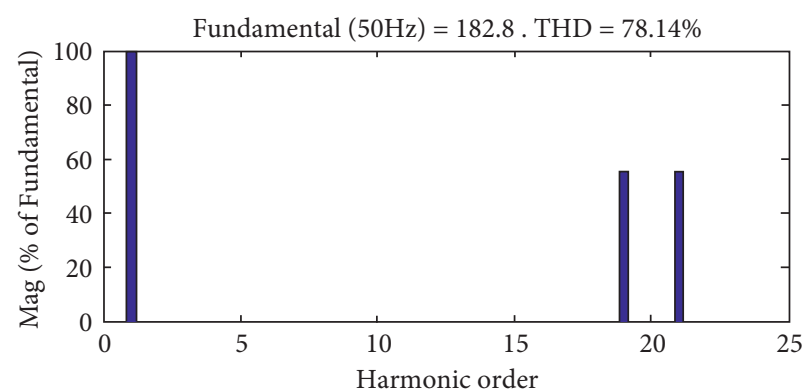

(b)

Figure 13: $(\mathrm{a}, \mathrm{b})$ Output voltage and harmonic spectrum for $\delta=101.454$.

TABLE 2: Comparison of individual harmonics as $\%$ of the fundamental voltage.

\begin{tabular}{|c|c|c|}
\hline \multirow{2}{*}{ Harmonic order } & \multicolumn{2}{|c|}{ Fundamental RMS output voltage } \\
\hline & Theoretical & Simulation \\
\hline$h_{1}$ & $39.8411(100 \%)$ & $39.98(100 \%)$ \\
\hline$h_{3}$ & 0 & 0.15918 \\
\hline$h_{5}$ & 0 & 0.035373 \\
\hline$h_{7}$ & 0 & 0.035373 \\
\hline$h_{9}$ & 0 & 0.141493 \\
\hline$h_{11}$ & 0 & 0.106118 \\
\hline$h_{13}$ & 0 & 0.070745 \\
\hline$h_{15}$ & 0 & 0.053059 \\
\hline$h_{17}$ & 0 & 0.15918 \\
\hline$h_{19}$ & 95.13678 & 93.95075 \\
\hline$h_{21}$ & 95.13678 & 94.00383 \\
\hline$h_{23}$ & 0 & 0.141493 \\
\hline$h_{25}$ & 0 & 0.053059 \\
\hline
\end{tabular}


TABLE 3: Comparison between SAC and AEM in terms of power factor.

\begin{tabular}{lcccc}
\hline Target output voltage & SAC & \multicolumn{2}{c}{ AEM } & THD \\
\hline 20 & THD & PF & 208.22 & 0.432922 \\
40 & 302.52 & 0.313854 & 185.18 & 0.475159 \\
60 & 211.03 & 0.428220 & 154.3 & 0.54386 \\
80 & 164.39 & 0.519706 & 126.12 & 0.621295 \\
100 & 133.88 & 0.598429 & 105.27 & 0.688726 \\
120 & 112.39 & 0.664727 & 81.14 & 0.749411 \\
130 & 93.91 & 0.728955 & 74.09 & 0.776532 \\
140 & 86.36 & 0.756837 & 61.12 & 0.803496 \\
160 & 78.81 & 0.785407 & 47.69 & 0.853248 \\
180 & 64.76 & 0.839363 & 30.39 & 0.902612 \\
200 & 51.66 & 0.88845 & 0.956793 \\
210 & 37.64 & 0.935898 & & 0.979916 \\
\hline
\end{tabular}

TABLE 4: Comparison of SAC and AEM in terms of harmonic distortion factor.

\begin{tabular}{lcc}
\hline Harmonic order & SAC & AEM \\
THD
\end{tabular}

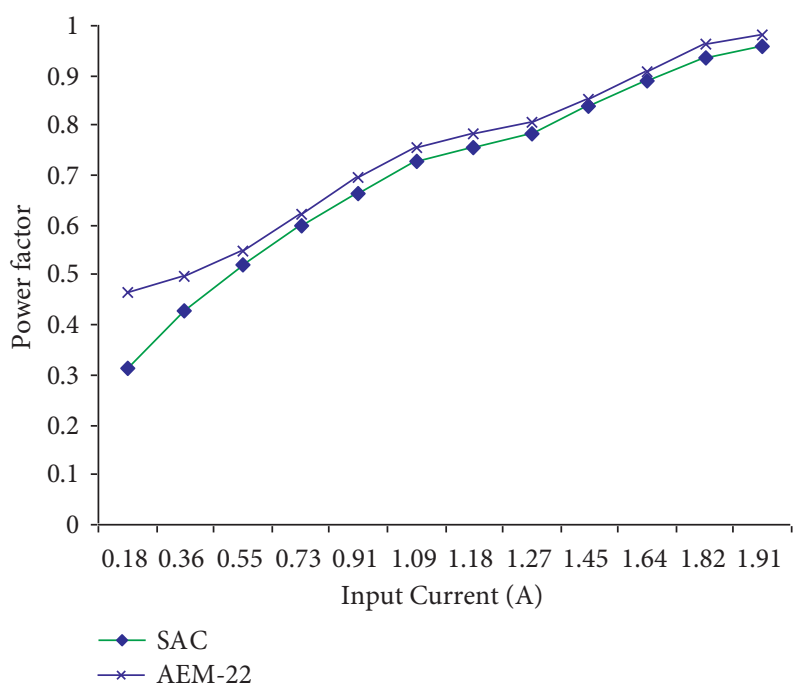

Figure 14: Variation of power factor with the input current. 


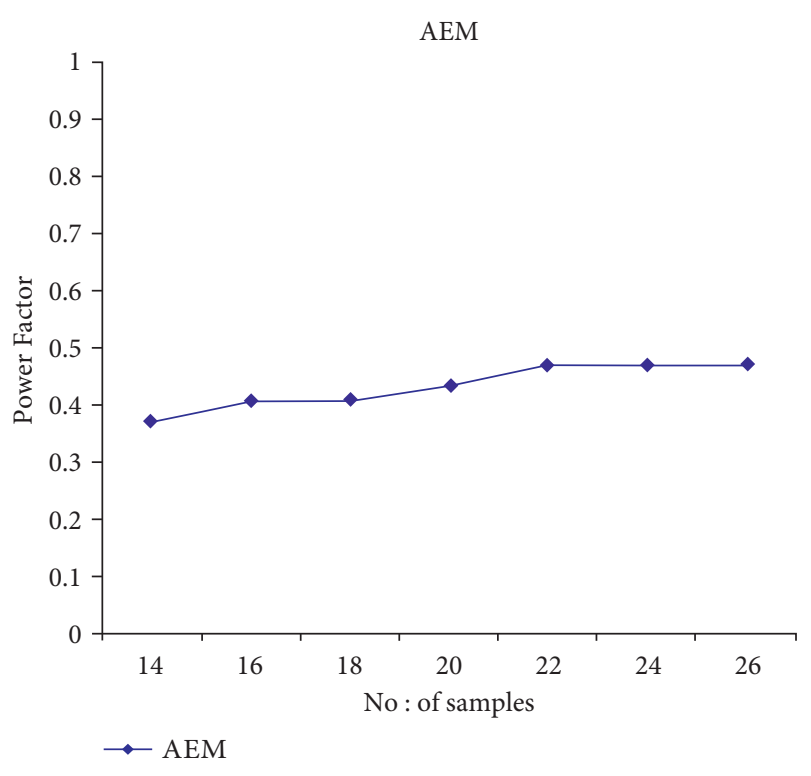

FIgURE 15: Variation of power factor with " $\mathrm{m}$ ".

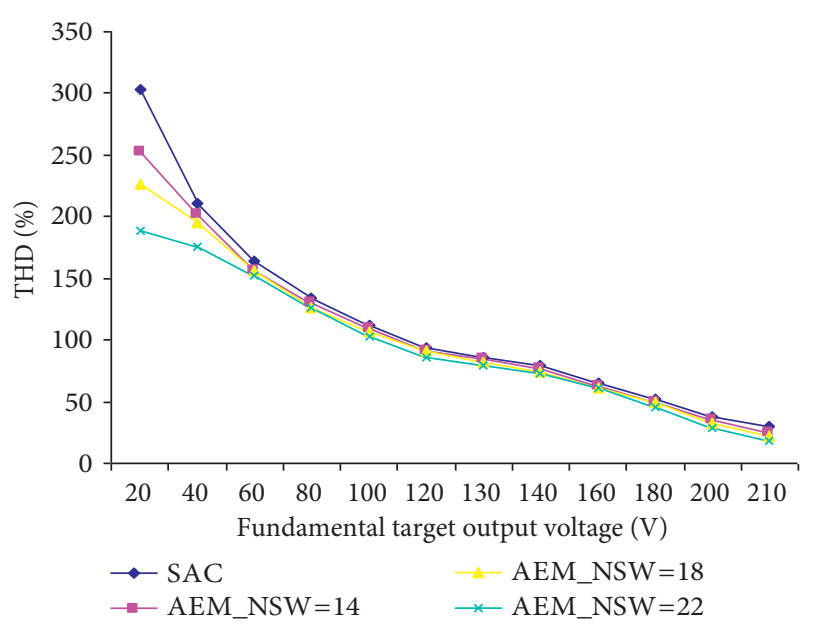

FIGURE 16: Variation of THD with the fundamental target output voltage.

\section{Conclusion}

The conventional forced commutated SAC method suggested fails to improve the power factor. It is seen that the total harmonic distortion is high at low output voltage. AEM employing the forced commutation technique improves the input PF considerably and improves the harmonic spectrum. The proposed method provides good AC power control. The output voltage fundamental component and the duty cycle of the control circuit are flatly linear; hence, a good linear voltage control is seen. The lower order harmonic components, which increase the filter size and cost, have been drastically reduced, besides having contributed to an enhancement in input power factor and THD.

\section{Data Availability}

The data used to support the findings of this research are available from the corresponding author upon request.

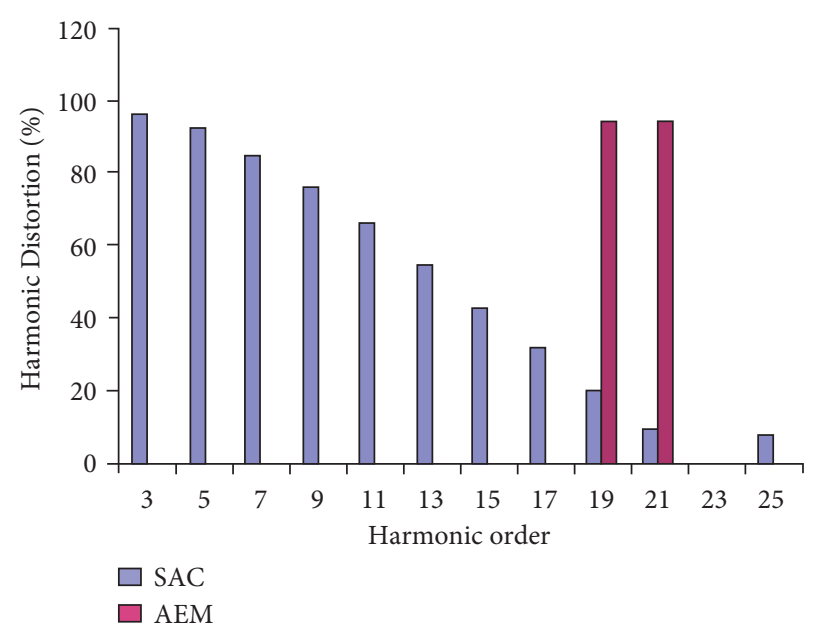

Figure 17: Comparison of individual harmonics order for a target output of $40 \mathrm{~V}$.

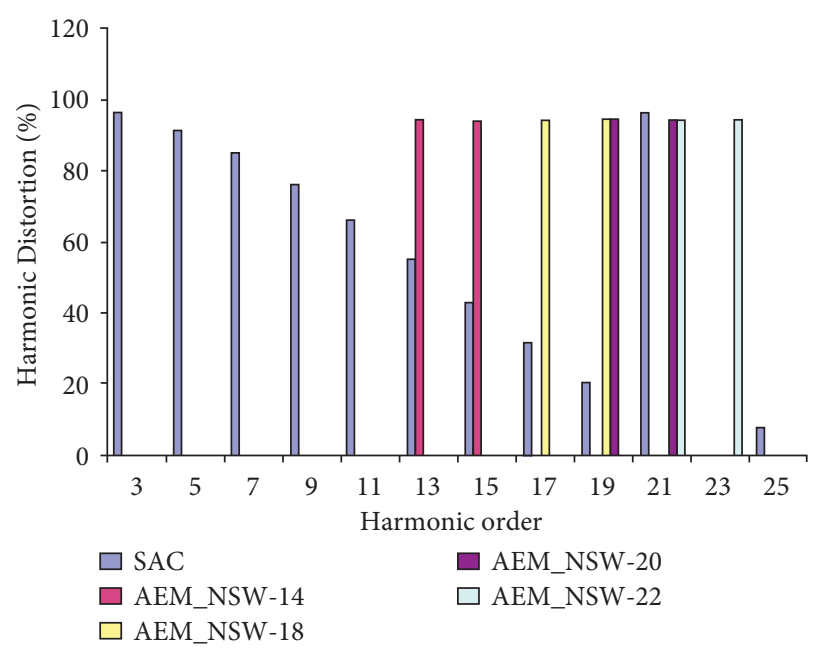

FIGURE 18: Variation in the individual harmonic order with increasing " $\mathrm{m}$ ".

\section{Conflicts of Interest}

The authors declare that they have no conflicts of interest.

\section{References}

[1] S. Williamson, "Reduction of the voltage and current harmonics introduced by a single- phase triac ac controller," Instrumentation, vol. 28, no. 4, 1981.

[2] E. El-Bidweihy, "Power factor of ac controllers for inductive loads," IEEE Transactions on Industrial Electronics and Control Instrumentation, vol. 27, no. 3, 1980.

[3] M.-Ho Park, "An improved PWM technique for ac choppers," IEEE Transactions on Power Electronics, vol. 4, no. 4, pp. 496-405, 1989.

[4] Do-H. Jang, "A new APWM technique with harmonic elimination and power factor control in AC choppers," in Proceedings of International IEEE conference, pp. 252-258, San Diego, CA, USA, November 1992.

[5] K. E. Addoweesh, "Microprocessor based harmonic elimination in chopper type ac voltage regulators," IEEE Transactions on Power Electronics, vol. 5, pp. 191-200, 1990. 
[6] F. Swift, "A new Walsh domain technique of harmonic elimination and voltage control in pulse-width modulated inverters," IEEE Transactions on Power Electronics, vol. 8, no. 2, pp. 170-185, 1993.

[7] T. Kato, "Sequential homotopy -based computation of multiple solutions for selected harmonic elimination in PWM inverters," IEEE Transactions on Circuits and Systems -1: Fundamental Theory and Applications, vol. 46, pp. 586-593, 1999.

[8] P. N. Enjeti, "Programmed PWM techniques to eliminate harmonics: a critical evaluation," IEEE Transactions on Industry Applications, vol. 26, pp. 302-316, 1990.

[9] H. S. Patel, "Generalized harmonic elimination and voltage control in thyristors inverters: part I-harmonic elimination," IEEE Transactions on Industry Applications, vol. 9, pp. 310317, 1973.

[10] H. S. Patel, "Generalized harmonic elimination and vol tage control in thyristors inverters: part II-voltage control technique," IEEE Transactions on Industry Applications, vol. 10, pp. 666-673, 1974.

[11] R. Corapsiz, H. Kahveci, and M. F. Çorapsiz, "Design and Implementation of Single-phase AC Voltage Controller with Phase Triggering Control," in 3rd International Conference on Advanced Engineering Technologies, Bayburt, September 2019.

[12] A.. Altintas, "A study on power quality improvement in PWM controlled AC voltage controller," Süleyman Demirel Üniversitesi Fen Bilimleri Enstitüsü Dergisi.vol. 12, 2009.

[13] F. M. Serra, L. M. Fernández, O. D. Montoya, W. G. González, and J. C. Hernández, "Nonlinear voltage control for threephase DC-AC converters in hybrid systems: an application of the PI-PBC method," Electronics, vol. 9, no. 5, p. 847, 2020.

[14] Md. R. Islam, Md Uddin, Md Uddin, I. Ahmed, and Md. M. Hasan, A Model of Voltage Stabilization Using PWM Inverter at the Load End, , pp. 154-159, IEEE, 2020.

[15] A. Viswas, C. Santhanam, and Y. Kashyap, "A single phase modular multilevel inverter based on controlled DC-cells under two SPWM techniques for renewable energy applications," in International Conference -RARE 2020 Inverter and PWM Control Technique suitable for Renewable Energy Applications, vol. 1, pp. 117-122, 2020.

[16] Prajakta Kasulakar, Chetan Dethe, Paras Patil, and Pratik Murkute, "Single phase inverter using pwm technique," International Journal of Engineering Applied Sciences and Technology, vol. 04, pp. 225-230, 2020. 